\title{
FIVE-DIMEISIONAL, QUASISPIN TOWARD A COMPLETE CLASSIFICATION OF THE ISOSPIN CHARACTERISTICS OF SHELL-MODEL STATES IN THE SENIORITY SCHEME
}

\author{
R. P. HEMENGER ${ }^{\dagger}$ and K. T. HECHT ${ }^{\dagger} \uparrow$ \\ Physics Department, The Universiry of Michigan, Ann Arbor, Michigan \\ Received 20 October 1969
}

\begin{abstract}
A solution is proposed for the inner multiplicity problem associated with the five-dimensional quasispin description of shell-model statcs. A classification scheme, in terms of an orthonormal basis, leads to tractable tisults with detinitc symmetry propertics under particle-hole conjugation. Explicit constructions are giver for the $R(5)$ irreducible representations $\left(\omega_{1} 1\right),\left(\omega_{1} \frac{3}{2}\right)$, $(t-1, t)$ for which the inner multiplicities are never greater than two. For siates of seniority $t=2$, reduced isosnin $t=1$, of a configuration $j^{n}$ gencral expressions are given for the matrix elemerts of an arbitrary two-body interaction, to determine their $n, T$ dependence, and to isolate those features of the actual interaction among nuclcons which are most cffective in splitting the isospin degeneracy of such states.
\end{abstract}

\section{Introduction}

For configurations of both neutrons and protons the ñve-dimensional quasispin formalism ${ }^{1-6}$ ) gives the best description of the isospin structure of shell-model states in the seniority scheme. The five-dimensional quasispin formalism also provides a natural tool for extracting the $n, T$ dependent factors of all nuclear matrix elements ( $n=$ nucleon number, $T=$ isospin $)^{7}$ ). By identifying the irreducible tensor character of operators under the quasispin group $R(5)$ and applying the Wigner Eckart theorem in quasispin space, it is possible to give reduction formulate which give matrix elements for the states of the coniguration $j^{n}$ in terms of the corresponding matrix elements for the configuration $j^{\nu}$. Although the explicit $n, T$ dependent factors of nuclear matrix elements have been derived by this technique for seniorities $t$ and reduced isospin $t$ corresponding to the simpler representations of $R(5)$ [ref. $\left.{ }^{8}\right)$ ], no general solution to this problem has been found because of the inner multiplicity problem associated with the quasispin group $\mathrm{R}(5)$. The five-dimensional quasispin description of shell-model states is plagued by the problem of a "missing" quantum number. The irreducible representation labcls of $R(5)$ are characterized by the seniority number $v$ and reduced isospin $t$. Besides ihese, four additional quantum numbers are needed to completely specify a basis state of a given irreducible rep-

† Present Address: Physics Department, Tuskogec Institute, Tuskegcc, Alabama.

tt Supported by US Office of Naval Research, Contract Nonr 1224(59). 
resentation. Of these four. the physically relevant or natural quantum numbers are $n$. $T$. and $M_{\tau}$. Unfortunately no simple fourth operator exists which commutes with the number operator. the operators $T^{2}$ and $T_{0}$, as well as with the Casimir invariants of $\mathrm{R}(5)$. For fixed $n, T, M_{T}$, therefore, there is in general some number, $m$, of indcpendent states in a given irreducible representation of $R(5)$, corresponding to $m$ difierent values of a Sour!h quantum number. Such states will be said to have a "T-multiplicity of $m$ ". So far derivations of the $n, r$ dependent factors of nuclear matrix elements in gencral algebraic form has been restricted to statcs with no such inner multiplicities ${ }^{8}$ ), that is 10 siates with "T-multiplicitics of $1 "$. Although these include some of the low-seniority states of greatest physical interest, an extension to more complicated $R(5)$ representations is needed to makc it possible to give the $n, T$ dependent factors for all matrix elements of actual practical interest in shellmodel calculations based on the seniority scheme.

A complete specification of the states with $T$-multiplicities greater than one can be given in terms of arbitrary labeling schemes. One such scheme was first suggested by Racah ${ }^{9}$ ). States of a given $t, t, n$. $T$ are built naturally from onc group of $t$ nucleons entircly free of pairs coupled to angular momentum $J=0$ which are coupled to reduced isospin $t$; and another group of nucleons made up of $p=\frac{1}{2}(n-v)$ pairs of nucleons, each coupled to $J=0, T=1$. These $p$ pairs are coupled to isospin $T_{p}$, where $T_{p}=p, p-2, p-4, \ldots$, and where the total isospin is the result of the vector coupling $T=T_{p}+t$. Racah suggested that the labei $T_{p}$, which has some physical significance, be used to distinguish the independent states of a given $v, t, n, T$. Since the labels $T_{p}$ are not related to the eigenvalues of a hermitcan opcrator, however, two states with different values of $T_{p}$ are in general not orthogonal to each other. Although there may be some merit in using a nonorthogonal basis, (see for example the remarks of Racah in ref. $\left.{ }^{10}\right)$ ); it is difficult to apply the formalism of the irreducible tensor calculus in such a basis. For purposes of extracting the $n, T$ dependent factors of nuclear matrix elcments. therefore, it becomes important to find a useiful orthogonal basis and a solution to the problem of the fourth operator. The analogous problem of a "missing" quantum number has been discussed in some detail by Racah ${ }^{10}$ ) for the group $S U(3)$ resiricted to the subgroup $R(3)$ generated by the three-dimensional orbital angular momentum operators. Racah shows that the missing opcrator can be expressed entirely in terms of two operators of degree three and four, respectively, in the infinitesimai operators which generate the full groun in this case. However, Racah was unable to find a function of thest two operators with rational eigejvalues.

Alternate labeling schemes for the five-dimensional quasispin group have been suggested on physical grounds. Since four particle correlations, involving alpha-like groupings of tour nucleons coupled to $J=0, T=0$, are important in nuclei, Parikh ${ }^{2}$ ) and Flowers and Szpikowski ${ }^{11}$ ) suggested that the states with $T$-multiplicitics greater than one be distinguished by their content of such "alpha-particle clusters". Flowers and Szpikowski, in particular, introduce an operator which "counis" the number 
of such four-particle clusters. It is ari operator of degree four in the infinitesimal operators of $\mathrm{R}(5)$. Unfortunately its eigenvalue structure is very complicated and its eigenvalues are in general not rational numbers. Goldberg ${ }^{12}$ ) and Groshen and Lipkin ${ }^{13}$ ) have also suggested a classification scheme based on the content of alphalike clusters. Their scheme classifies states according to the number of nucleons not members of $J=0, T=0$ quadruples. All these schemes, however, would lead to $\mathrm{R}(5)$ Wigner cocfficients of very complicated algcbraic siructure. They have an additional disadvantage, in that the states based on these schemes have no definite symmetry properties under particle-hole conjugation.

For these reasons a new classification scheme based on a somewhat different approach to the problem of the fourth operator will be proposed, with the idea that a useful basis must satisfy the following criteria.

(i) Its states must be members of an orthonormal set.

(ii) It must yield tractable results; that is, the eigenvalues of the fourth operator must be rational numbers, and the operator must be chosen such that the algebraic structure of the $R(5)$ Wigner coefficients is relatively simple.

(iii) It must have definite symmeiry propertics under particle-hole conjugation.

The search for such a basis applicable to all conceivable irreducible representations of $R(5)$ has not met with success. However, a much more limited approach will be sufficient for nuclear shell theory. Since only $R(5)$ irreducible representations with rather low $T$-multiplicities are of practical importance for shell-model calculations, it will be sufficient to consider only such cases. The main result of this investigation will be a complete classification of the basis states of all those $R(5)$ representations with $T$-multiplicities no greater than two accordirg to a scheme which satisfies the above critcria. For simple shells with $j \leqq \frac{\text { ? }}{2}$. (or for mixed configurations with $\left.\Omega=\Sigma\left(j+\frac{1}{2}\right) \leqq 5\right)$, this includes all possible $\mathrm{R}(5)$ representations. Out of the 28 possible $\mathrm{R}(5)$ representations in a $j=\frac{1}{2}$ - shell, for example, only one contains states with a $T$-multiplicity greater than two. Since it is feasible to apply the techniques developed in this work to special cases of representations containing states with $T$-multiplicities greater than iwo, the problem of finding a tractable classification scheme can be considered as solved.

The fourth operator needed for a complete classification of the states of $R(5)$ will commute with the operators, $T^{2}$, and $T_{0}$, the number operator, and the Casimiinvariants of $R(5)$ if it is an isoscalar operator, which conserves nucleoin number, and is built from the infinitesimal operators which generate $R(5)$. The latter include, besides the number operator and the isospin operators $T$, the operators which create and annihilate pairs of nucleons coupled to $J=0, T=1$ :

$$
\begin{aligned}
& A^{\dagger}\left(M_{\Gamma}\right)=\frac{1}{2} \sum_{m, m_{t}}\left\langle\frac{1}{2} m_{t} \frac{1}{2} m_{t}^{\prime} \mid 1 M_{T}\right\rangle(-1)^{j-m} a_{j m m_{t}}^{\dagger} a_{j-m m_{t}^{\prime}}^{\dagger}, \\
& A\left(M_{T}\right)=\left(A^{+}\left(M_{T}\right)\right)^{\dagger} .
\end{aligned}
$$


The fourth operaior is to be built from the operators

$$
O_{20}=\left(A^{\dagger} \cdot A^{\dagger}\right) \text { and } O_{-20}=(A \cdot A),
$$

where $\left(A^{\dagger} \cdot A^{\dagger}\right)$ and $(A \cdot A)$ arc scalar products (in isospin space) of the $J=0, T=1$ pair creation and annihilation operators. The operators $O_{20}, O_{-20}^{\circ}$ have the property that they create or annihilate, respectively, four nucleons coupled to $J=0, T=0$. (The subscript notation $O_{20}, O_{-20}$ will become clear in sect. 3.)

The anticommutator and the commutator of the operators $O_{20}$ and $O_{-20}$ form the potential building blocks for the fourth operator. The anticommutator of $\mathrm{O}_{20}$ and $O_{-20}$ is an operator of degree four in the infinitesimal operators of $\mathrm{R}(5)$, while the commutator reduces to the operator $T \cdot\left[A^{\dagger} \times A\right]$, (except for trivial functions of $T_{0}$ and the number operator; see table 8 of ref. ${ }^{8}$ ) or eq. $(39 \mathrm{c})$ of ref. $\left.{ }^{13}\right)$ ); that is, it is an operator of degree three in the infinitesimal operators. These two operators are the analogues of the operators of degree three and four-discussed by Racah in his search for the missing quantum number for the group chain $S U(3) \supset R(3)$. In the classification scheme to be used in this investigation the fourth operator is to be chosen as

$$
O_{y}=\left(O_{20} O_{-20}+O_{-20} O_{20}\right)+y\left(O_{20} O_{-20}-O_{-20} O_{20}\right)
$$

where $y$ is a function of the isospin and number operator, chosen such that the operator $O_{y}$ is invariant under particle-hole conjugation and that the resultant eigenstates are simplc enough to be useful in practical cases. If the function $y$ were replaced by the number $y=1$, the operator $O_{y}$, would collapse to that proposed by Flowers and Szpikowski ${ }^{11}$ ) as an operator which "counts" the number of four-particle clusters coupled to $J=0, T=0$. Howcver, since particle-hole conjugation is equivalent to complex conjugation in the fivc-dimensional quasispin space, and since the anticommutator and commutator of $O_{20}$ and $O_{-20}$ have the symmetry property + and - , respectively, under complex conjugation, the function $y$ must have the symmetry property - under such conjugation.

In sect. 2 the isospin structure of the $\mathrm{R}(5)$ irreducible representations with $T$ multiplicities not greater than two is discussed. A very brief review of notation is also given. In sect. 3 expiicit constructions are given of (i) the operators $O_{y}$ of eq. (3), and (ii) the ful! set of eigenstates for the representations of sect. 2 . The techniques used to calculate $\mathrm{R}(5)$ Wigner coefficients needed to extract the $n, T$, dependent factors of nuclear matrix elements are the same as those described in ref. ${ }^{8}$ ). The more basic of thesc coefficients are tabulated in general algebraic form in an appendix. In sect. 4 some applications are given. In particular, matrix elements are given in general algebraic form for the $v=2$ states of the pure configuration $j^{n}$ with a $T$ multiplicity of 2 for (i) the most general charge independent two-body interaction. and (ii) the Coulomb interaction, in an attempt to study those features of the actual interaction among nucleons which are most effective in splitting the isospin degeneracy of such states. 


\section{The irreducible representations with $T$-multiplicities of 2}

The notation will follow that of ref. ${ }^{8}$ ) so that only a very bricl review will be given. Table 1 shows the ten infinitesimal operators which generate the quasispin group $R(5)$. The two commuting operators which label the weights of this rank 2 group are $H_{1}=\frac{1}{2} N_{\mathrm{op}}-\left(j+\frac{1}{2}\right)$ and $H_{2}=T_{0}$. Under complex conjugation, (particlehole conjugation), the definition of $H_{1}$ leads to the symmetry $H_{1}$ cigen $\rightarrow-H_{1 \text { eigen }}$.

TABiE 1

The gencrators of $\mathrm{R}(5)$

\begin{tabular}{cc}
\hline Quasispin opcrators & Standard form \\
\hline$N_{0 D}-(j+1)$ & $H_{1}$ \\
$T_{0}$ & $H_{2}$ \\
$A^{\dagger}(1)$ & $E_{11}$ \\
$A(1)$ & $E_{-1-1}$ \\
$A^{+}(-1)$ & $-E_{1-1}$ \\
$A(-1)$ & $-E_{-11}$ \\
$A^{\dagger}(0)$ & $E_{10}$ \\
$A(0)$ & $E_{-10}$ \\
$T_{+}$ & $\sqrt{2} E_{01}$ \\
$T_{-}$ & $\sqrt{2} E_{0-1}$ \\
\hline
\end{tabular}

The irreducible representations are labeled by $\left(\omega_{1} \omega_{2}\right)$, the maximal wcight valucs based on the pair of operators $H_{1}, T_{0}$. These are related to the seniority number $v$ and reduced isospin $t$ by

$$
\omega_{1}=j+\frac{3}{2}-\frac{1}{2} v, \quad \omega_{2}=t
$$

(For mixed configurations the degencracy number $j+\frac{1}{2}$ is to be replaced by $\Omega=\Sigma\left(j+\frac{1}{2}\right)$.)

The $n, T$ structure of an arbitrary irreducible representation has been given by several techniques ${ }^{5,7,14}$ ). It can best be summariced by the following rales ${ }^{14}$ ). The allowed values of $H_{1}$ and $T$ in $\left(\omega_{1} t\right)$ are given by the possible angular momentum couplings $T=T_{p}+t$, where

(i) $T_{p}$ has the possible valucs $T_{p}=p^{\prime}, p^{\prime}-2, p^{\prime}-4, \ldots$, for $H_{1}= \pm\left|\omega_{1}-p^{\prime}\right|$, $p^{\prime}=0,1,2, \ldots\left(\leqq \omega_{1}\right)$. The allowed states are subject to the following restrictions:

(ii) $T \leqq \omega_{1}$.

(iii) If the possible couplings of $T_{p}+t$ lead to a state of specific $T$ more than once, a slate of specific $T$ occurs at most $q$ times, where $q=\min \left(\omega_{1}-T+1, \omega_{1}-t+1\right)$. Straightforward application of these rules shows that the representations $\left(\sigma_{1} 0\right)$, $\left((i), \frac{1}{2}\right)$, and $(t)$ have no states with $T$-multiplicities greater than one. These represen- 
tations have been discussed in detail in ref. $\left.{ }^{8}\right)$. It can further be seen that the representations $(\omega, 1),\left((1), \frac{3}{2}\right)$. and $(t+l t)$ have no states with $T$-multiplicitics greater than two. The full $I_{1}, T$ structure of these representations is illustrated in tables 2,3

TABLE 2

$H_{1}, \Gamma$ structure of representations $\left.\left(()_{1} 1\right)^{a}\right)$

\begin{tabular}{|c|c|c|c|c|c|c|c|}
\hline$\dot{H}$ & & & & & & $T$ & \\
\hline$\omega_{1}$ & $i$ & $1_{(2)}$ & & & & & \\
\hline$\omega_{1}-1$ & $0_{(1)}$ & $i_{(0)}$ & $2(2)$ & & & & \\
\hline$\omega_{1}-2$ & $\mid$ & $1_{(1,2)}^{2}$ & $2_{(0)}$ & $3(2)$ & & & \\
\hline$\omega_{1}-3$ & $0_{(1)}$ & $1_{(0)}$ & $2_{(1,2)}^{2}$ & $3(0)$ & $4_{(2)}$ & & \\
\hline . & $i$ & $\cdot$ & . & . & . & $\cdot$ & \\
\hline$\cdot$ & , & • & • & . & • & $\cdot$ & . \\
\hline . & $i$ & . & $\cdot$ & . & . & $\cdot$ & $\cdot \cdot$ \\
\hline 2 & $i$ & & & & $\cdot$ & $\cdot$ & $\cdot\left(\omega_{1}-2\right)_{(0)}\left(\omega_{1}-1\right)_{(2)}$ \\
\hline 1 & ? & & & & $\cdot$ & $\cdot$ & - $\left(\omega_{1}-2\right)_{(1,2)}^{2}\left(\omega_{i}-1\right)_{(0)}\left(\omega_{1}\right)_{(2)}$ \\
\hline 0 & ! & & & & . & $\cdot$ & $\cdot\left(\omega_{1}-2\right)_{(0)} \quad\left(\omega_{1}-i\right)_{(1,2)}^{2}\left(\omega_{1}\right)_{(0)}$ \\
\hline-1 & $!$ & & & & · & & - $\left(\omega_{1}-2\right)_{(1,2)}^{2}\left(\omega_{1}-1\right)_{(0)}\left(\omega_{1}\right)_{(2)}$ \\
\hline-2 & $\begin{array}{l}1 \\
1\end{array}$ & & & & $\cdot$ & $\cdot$ & $\cdot\left(\omega_{1}-2\right)_{(0)}\left(\omega_{1}-1\right)_{(2)}$ \\
\hline$\cdot$ & $!$ & · & · & • & · & $\cdot$ & $\cdot \cdot$ \\
\hline • & $i$ & • & $\cdot$ & • & · & $\cdot$ & \\
\hline$\cdot$ & $\vdots$ & $\cdot$ & . & . & . & - & \\
\hline$-\left(\omega_{1}-3\right)$ & $o_{(1)}$ & $1_{(0)}$ & $2_{(1,2)}^{2}$ & $3(0)$ & $4(2)$ & & \\
\hline$-\left(\omega_{1}-2\right)$ & 1 & $1_{(1,2)}^{2}$ & $2(0)$ & $3(2)$ & & & \\
\hline$-\left(\omega_{1}-1\right)$ & $O_{(1)}$ & $1_{(0)}$ & $2(2)$ & & & & \\
\hline$-\omega_{1}$ & 1 & $1_{(2)}$ & & & & & \\
\hline
\end{tabular}

2) The superseripts are the $T$-multiplicity. The subscripts are the possible values of the label $\beta$.

and 4. Explicit constructions are given in sect. 3 for the full sel of eigenstates for these three types of $\mathrm{R}(5)$ representations. The $\mathrm{R}(5)$ Wigner coefficients involving these represcntations are calculated, making it possible to give the $n, T$ dependent factors of nuclear matrix elements for all cases with $t \leqq \frac{3}{2}$ or ()$_{1}=t+1$. 
TABLB 3

$I_{1}, \Gamma$ structure of represintations $\left(\left(b_{1}, 3^{2}\right)^{2}\right)$

\begin{tabular}{|c|c|c|c|c|c|c|c|}
\hline$H_{1}$ & & & & $-\ldots$ & $T$ & & - \\
\hline$\omega_{1}$ & & $\left(\frac{3}{2}\right)_{(2)}$ & & & & & \\
\hline$\omega_{1}-1$ & $\left(\begin{array}{l}1 \\
2\end{array}\right)_{(1)}$ & $\left(\frac{3}{2}\right)_{(-2)}$ & $\left(\frac{5}{2}\right)_{(2)}$ & & & & \\
\hline$\omega_{1}-2$ & $\left(\frac{1}{2}\right)(-1)$ & $\left(\frac{3}{2}\right)_{(1,2)}^{2}$ & $\left(\frac{5}{2}\right)_{(-2)}$ & $\left(\begin{array}{l}7 \\
2\end{array}\right)_{(2)}$ & & & \\
\hline$\omega_{1}-3$ & $\left(\begin{array}{l}1 \\
2\end{array}\right)_{(1)}$ & $\left(\frac{3}{2}\right)_{(-1,-2)}^{2}$ & $\left(\begin{array}{l}5 \\
2\end{array}\right)_{(1,2)}^{2}$ & $\left(\begin{array}{l}7 \\
2\end{array}\right)(-2)$ & $\left(\begin{array}{l}9 \\
2\end{array}\right)_{(2)}$ & & \\
\hline · & . & · & $\cdot$ & · & . & • & \\
\hline · & · & · & • & · & · & · & $\cdot$ \\
\hline . & · & · & · & · & · & $\cdot$ & $\cdot \cdot$ \\
\hline$\frac{5}{2}$ & & & & & - & $\cdot$ & $\cdot\left(\omega_{1}-2\right)_{(-2)}\left(\omega_{1}-1\right)_{(2)}$ \\
\hline$\frac{3}{2}$ & & & & & . & . & $\cdot\left(\omega_{1}-2\right)_{(1,2)}^{2} \quad\left(\omega_{1}-1\right)_{(-2)} \quad\left(\omega_{1}\right)_{(2)}$ \\
\hline$\frac{1}{2}$ & & & & & · & • & $\cdot\left(\omega_{1}-2\right)_{(-1,-2)}^{2}\left(\omega_{1}-1\right)_{(1,2)}^{2} \quad\left(\omega_{1}\right)_{(-2)}$ \\
\hline$-\frac{1}{2}$ & & & & & - & · & $\left(\omega_{1}-2\right)_{(1,2)}^{2} \quad\left(\omega_{1}-1\right)_{(-1,-2)}^{2}\left(\omega_{1}\right)_{(2)}$ \\
\hline$-\frac{3}{2}$ & & & & & - & · & - $\left(\omega_{1}-2\right)_{(-1,-2)}^{2}\left(\omega_{1}-\hat{1}\right)_{(2)} \quad\left(\omega_{1}\right)_{(-2)}$ \\
\hline$-\frac{5}{2}$ & & & & & . & - & $\cdot\left(\omega_{1}-2\right)_{(2)} \quad\left(\omega_{1}-1\right)_{(-2)}$ \\
\hline . & . & · & $\cdot$ & · & - & $\cdot$ & $\cdot \cdot$ \\
\hline · & . & · & · & $\cdot$ & $\cdot$ & - & . \\
\hline - & . & - & · & $\cdot$ & $\cdot$ & $\cdot$ & \\
\hline$-\left(\omega_{1}-3\right)$ & $\left(\frac{1}{2}\right)_{(-1)}$ & $\left(\frac{3}{2}\right)_{(1,2)}^{2}$ & $\left(\frac{5}{2}\right)_{(-1,-2)}^{2}$ & $\left(\frac{7}{2}\right)_{(2)}$ & $\left(\frac{9}{2}\right)_{(-2)}$ & & \\
\hline$-\left(\omega_{1}-2\right)$ & $\left(\begin{array}{l}1 \\
2\end{array}\right)_{(1)}$ & $\left(\frac{3}{2}\right)_{(-1,-2)}^{2}$ & $\left(\frac{5}{2}\right)_{(2)}$ & $\left(\frac{7}{2}\right)_{(-2)}$ & & & \\
\hline$-\left(\omega_{1}-1\right)$ & $\left(\begin{array}{l}1 \\
2\end{array}\right)(-1)$ & $\left(\frac{3}{2}\right)_{(2)}$ & $\left(\begin{array}{l}5 \\
2\end{array}\right)(-2)$ & & & & \\
\hline$-\omega_{1}$ & & $\left(\frac{3}{2}\right)_{(-2)}$ & & & & & \\
\hline
\end{tabular}

1) The superseripts are the $T$-multiplicity. The subscripts are the possible values of the label $\beta$. 
TABLE 4

$H_{1}, T$ structure of representations $\left.(t+1, t)^{\star}, \mathrm{b}\right)$

\begin{tabular}{|c|c|c|c|c|c|c|c|c|c|}
\hline$H_{1}$ & & & & & & $T$ & & & \\
\hline$t+1$ & & & & & & & & $t_{(1)}$ & \\
\hline$t$ & & & & & & & $(t-1)_{(1)}$ & $t_{(1)}$ & $(t+1)_{(2)}$ \\
\hline$t-1$ & & & & & & $(t-2)_{(1)}$ & $(t-1)_{(t)}$ & $t_{(1,2)}^{2}$ & $(t+1)_{(2)}$ \\
\hline$t-2$ & & & & & $(t-3)_{(1)}$ & $(t-2)_{(1)}$ & $(t-1)_{(1,2)}^{2}$ & $t_{(1,2)}^{2}$ & $(t+1)_{(2)}$ \\
\hline . & & & & & . & • & · & . & . \\
\hline . & & & & . & . & . & . & . & . \\
\hline . & & & . & $\cdot$ & . & . & . & . & . \\
\hline 2 & & $1_{(1)}$ & $2(1)$ & $\cdot \cdot$ & - & & & & \\
\hline 1 & $0_{(1)}$ & $I_{(1)}$ & $2_{(1,2)}^{2}$ & . . & . & & & & \\
\hline 0 & & $1_{(1,2)}^{2}$ & $2_{(1,2)}^{2}$ & . . & . & & & & \\
\hline-1 & $0_{(1)}$ & $1_{(1)}$ & $2_{(1,2)}^{2}$ & · & . & & & & \\
\hline-2 & & $1_{(1)}$ & $2(1)$ & . . & - & & & & \\
\hline . & & & · & $\cdot \cdot$ & - & . & . & . & . \\
\hline$\cdot$ & & & & $\cdot \cdot$ & $\cdot$ & $\cdot$ & · & · & $\cdot$ \\
\hline$\cdot$ & & & & . & . & • & . & • & . \\
\hline$-(t-2)$ & & & & & $(t-\hat{3})_{(1)}$ & $(t-2)_{(1)}$ & $(t-1)_{(1,2)}^{2}$ & $t_{(1,2)}^{2}$ & $(t+1)_{(2)}$ \\
\hline$-(t-1)$ & & & & & & $(t-2)_{(1)}$ & $(t-1)(1)$ & $t_{(1,2)}^{2}$ & $(t+1)_{(z)}$ \\
\hline$-t$ & & & & & & & $(t-1)_{(1)}$ & $t_{(:)}$ & $(t+1)_{(2)}$ \\
\hline$-(t+1)$ & & & & & & & & $t_{(1)}$ & \\
\hline
\end{tabular}

$\left.{ }^{2}\right) t$ may be integer or half-odd integer. In the latter case both $T$ and $H_{1}$ arc of course also haliodd integer so that the $H_{1}, T$ structure will differ slightly from what is shown in the left ceittral portion of the above table. The precise structure in any case can be found by using the threc rules stated in sect. 2.

b) The superscripts are the $T$-multiplicity. 'The subscripts are the possible valucs of the iabel $\beta$.

\section{Explicit construction of $T$-multiplicity 2 states}

Basis states of irreducible representations of $R(5)$ require six labels, two of which are the representation labels. These states will be written $\left|\left(\omega_{1} t\right) \beta H_{1} T M_{T}\right\rangle$ where $\beta$ is the label replacing the fourth or "missing" quantum number. Only representations having states with $T$-multiplicity 2 but no states with $T$-multiplicitics greater than 2 are to be considered. Thus if $\omega_{1}, t, H_{1}, T$, and $M_{T}$ are fixed, $\beta$ can take no more than two values. 
The explicit construction is to be carried oul by the use of step-operators built from the infinitesimal generators, $E_{a b}$. The full set of basis states for a given $\left(\omega_{1} t\right)$ is then obtained by successive application of these step-operators to one of the states of maximal weight. The maximal weight states which have led to the simplest construction are the so-called "corner states". The normalized "corner state" for an irreducible representation $\left(\omega_{1} t\right)$ is defined by

$$
\mid \text { c.st. }\rangle=\left|(\omega, t) \beta=2, H_{1}=t, T=M_{r}=\omega_{1}\right\rangle .
$$

(Note its location in one of the corners of tables $2,3,4$.) This is always a state of $T$ mulitiplicity 1 so that there are no other values of $\beta$ corresponding to these vaiues of $H_{1}$ and $T$. The relevaint operators for the construction of states are,

$$
\begin{aligned}
O_{+1-1} & =E_{0-1}^{2} E_{11}-E_{0-1} E_{10}\left(2 T_{0}+1\right)-E_{1-1} T_{0}\left(2 T_{0}+1\right), \\
O_{-1-1} & =E_{0-1}^{2} E_{-11}-E_{0-1} E_{-10}\left(2 T_{0}+1\right)-E_{-1-1} T_{0}\left(2 T_{0}+1\right), \\
O_{10} & =-E_{0-1} E_{11}+E_{10}\left(T_{0}+1\right)-E_{1-1} E_{01}, \\
O_{-10} & =-E_{0-1} E_{-11}+E_{-10}\left(T_{0}+1\right)-E_{-1-1} E_{01}, \\
O_{20} & =2 E_{1-1} E_{11}+E_{10}^{2}, \\
O_{-20} & =2 E_{-1-1} E_{-11}+E_{-10}^{2} .
\end{aligned}
$$

Here the $E_{a b}$ are the group generators in standard form (see table 1). The first subscript is the change in the eigenvalue $H_{3}$ resulting from opcrating on an $\mathrm{R}(5)$ state. The second is the change in the eigenvalue $M_{T}$. The operators $O_{ \pm 10}, O_{ \pm 20}$ are isoscalars so that they leave $T$ unchanged, $O_{ \pm 1},-1$ on the other hand when operating on a state with $M_{T}=T$ decrease both $T$ and $M_{T}$ by unity.

\subsection{THE REPRESENTATION $\left(\omega_{1} 1\right)$}

There are two essentially different classes of basis states in this representation.

(i) Those states with $\omega_{1}-T-H_{1}=$ even integer have a $T$-multiplicity of 1 and will be labeled $\beta=0$ (sce table 2 ). These states were constructcd in ref: ${ }^{8}$ ) where the labei $\kappa=0$ was used.

(ii) Those states with $\omega_{1}-T-H_{1}=$ odd integer include all the $T$-muitiplicity 2 states and are labeled $\beta=1,2$. Two independent basis states must be constructed for each $H_{1}$ and $T$. One way of doing this is 10 put,

$$
\begin{aligned}
& \left.|a ; p q\rangle=O_{+1-1}^{p} O_{-1-1}^{q+1} \mid \text { c.st. }\right\rangle \\
& \left.|b ; p q\rangle=O_{+1-1}^{p+1} O_{-1-1}^{q} O_{-20} \mid \text { c.st. }\right\rangle .
\end{aligned}
$$

Here, $H_{1}=p-q$, and $T=\left(\omega_{1}-1-p-q\right.$. The effect of the operator $O_{y}$ (eq. (3)) on these states can be discovered by commuting it through to the corner state. Diagonalizing $O_{y}$ in this $2 \times 2$ system gives,

$$
O_{,}\left\{|a ; p q\rangle+\sigma_{ \pm}|b ; p q\rangle\right\}=i_{ \pm}\left\{|a ; p q\rangle+\sigma_{ \pm}|b ; p q\rangle\right\}
$$


where,

$$
\begin{aligned}
2(p+1)\left(2 \omega_{1}+1-2 p+2 y\right) \sigma_{ \pm}=-\left\{y+(p-q-y)\left(2 \omega_{1}+3\right)\left(2 \omega_{1}-1-2 p-2 q\right)\right\} \\
\quad \pm\left\{\left[y+(p-q-y)\left(2 \omega_{1}+3\right)\left(2 \omega_{1}-1-2 p-2 q\right)\right]^{2}\right. \\
\left.+4(p+1)(q+1)\left(2 \omega_{1}+1-2 q-2 y\right)\left(2 \omega_{1}+1-2 p+2 y\right)\right\}^{1},
\end{aligned}
$$

with eigenvalues,

$$
\begin{aligned}
\dot{\lambda}_{ \pm}=1+ & 2(p+1)(q+1)\left(2 \omega_{1}+1-2 q\right)\left(2 \omega_{1}+1-2 p\right) \\
& +[(p-q) y-1]\left[\left(2 \omega_{1}+3\right)\left(2 \omega_{1}-1-2 p-2 q\right)+8(p+1)(q+1)\right] \\
& \pm\left\{\left[y+(p-q-y)\left(2 \omega_{1}+3\right)\left(2 \omega_{1}-1-2 p-2 q\right)\right]^{2}\right. \\
& \left.+4(p+1)(q+1)\left(2 \omega_{1}+1-2 q-2 y\right)\left(2 \omega_{1}+1-2 p+2 y\right)\right\}^{\frac{1}{2}} .
\end{aligned}
$$

A choice of $y$, which leads to rational $\sigma_{ \pm}$and preserves particle-hole symmetry (see the intı oduction) is

$$
y\left(H_{1} T\right)=H_{1} \frac{2 T+1}{7+1} \cdot\left(=-y\left(-H_{1} T\right)\right)
$$

The normalized eigenstates of $O_{y}$ with this choice for $y$ are

$$
\begin{aligned}
& \left|\left(\omega_{1} 1\right) \beta=1, H_{1}=p-q, T=M_{T}=\omega_{1}-1-p-q\right\rangle=N_{1}(p q)[-|a ; p q\rangle+|b ; p q\rangle], \\
& \left|\left(\omega_{1} 1\right) \beta=2, H_{1}=p-q, T=M_{T}=\omega_{1}-1-p-q\right\rangle \\
& \quad=N_{2}(p q)\left\{\left(\omega_{1}+1\right) \stackrel{\left[\left(\omega_{1}-p-q\right)\left(2 \omega_{1}+1-p-q\right)+(p-q)\left(\omega_{1}-1-p-q\right)\right]}{(q+1)\left(2 \omega_{1}+1-p-q\right)}|a ; p q\rangle\right. \\
& \left.\quad+\left(\omega_{1}+1\right) \stackrel{\left[\left(\omega_{1}-p-q\right)\left(2 \omega_{1}+1-p-q\right)-(p-q)\left(\omega_{1}-1-p-q\right)\right]}{(p+1)\left(2 \omega_{1}+1-p-q\right)}|b ; p q\rangle\right\},
\end{aligned}
$$

where

$$
\begin{aligned}
& N_{1}(p q)=(-1)^{p+q} \\
& \times\left\{\begin{array}{r}
-\overline{\langle a ; p q| b} ; p q\rangle\left(\omega_{1}+1\right) \\
\quad \times\left[\left(\omega_{1}-p-q\right)\left(2 \omega_{1}+1-p-q\right)(p+q+2)+\left(\omega_{1}-1-p-q\right)(p-q)^{2}\right]
\end{array}\right\} \\
& N_{2}(p q)=N_{1}(p q) \\
& \times\left\{\begin{array}{c}
(--1)(q+1)\left(2 \omega_{1}+1-p-q\right) \\
\left(\left(\omega_{1}+1\right)\left(\omega_{1}+2\right)\left(\omega_{1}-1-p-q\right)\left(\omega_{1}-p-q\right)\left(2 \omega_{1}+1-2 p\right)\left(2 \omega_{1}+1-2 q\right)\right.
\end{array}\right\}^{\frac{1}{2}}, \quad(10 a)
\end{aligned}
$$

$\langle a ; p q \mid b ; p q\rangle$

$$
\begin{aligned}
=-\overline{2^{2 p+}} \frac{(p+1) !(q+1) ![}{\omega_{1}} \frac{\left.\left(2 \omega_{1}+1\right) !\right]^{3}\left(\omega_{1}-p\right) !\left(\omega_{1}-q\right) !\left(2 \omega_{1}+1-p-q\right)}{\left(2 \omega_{1}+1-2 p\right) !} & \left.-2 \omega_{1}+1-2 q\right) ! \\
& \times\left(\omega_{1}-1-p-q\right) !\left(\omega_{1}-p-q\right) !\left(2 \omega_{1}+1-2 p-2 q\right) !
\end{aligned}
$$




\subsection{THE REPRESENTATIONS $\left(\omega_{1} 3\right)$}

There are two classes of states in this representation which are essentially different, each of which contains states of $T$-multiplicity 2 . Under particle-hole conjugation cach state in one class goes into a corresponding state in the other.

(i) For those states with $\omega_{1}+\frac{3}{2}-T-H_{1}=$ cven integer the labels $\beta=1,2$ will be used (see table 3). For given $H_{1}, T$ define the two independent states,

$$
\begin{aligned}
& \left|a^{(+)} ; p q\right\rangle=O_{+1-1}^{p} O_{-1-1}^{q+1}|c . s t .\rangle, \\
& \left.\left|b^{(+)} ; p q\right\rangle=O_{+1-1}^{p+1} O_{-1-1}^{q} O_{-20} \mid \text { c.st. }\right\rangle,
\end{aligned}
$$

where $H_{1}=p-q+\frac{1}{2}, T=\omega_{1}-1-p-q ; O_{y}$ is again to be diagonalized in this $2 \times 2$ system. A choice of $y$ which makes the eigenstates of $O_{y}$ simple is,

$$
y^{(+)}\left(H_{1} T\right)=-\frac{\left(H_{1}-\frac{1}{2}\right)}{\left.\left(2 \omega_{1}+3\right)(2 T+1)-4 T\right]-\left(2 \omega_{1}+1\right)\left(\omega_{1}+1-T\right)}\left(\frac{-1}{\left(2 \omega_{1}+3\right)(2 T+1)+4\left(\omega_{1}-T\right)} .\right.
$$

The resulting normalized eigenstates are,

$$
\begin{aligned}
& \left|\left(\omega_{1} \frac{3}{2}\right) \beta=1, H_{1}=p-q+\frac{1}{2}, T=M_{T}=\omega_{1}-1-p-q\right\rangle \\
& =N_{1}^{(+)}(p q)\left[-\left|a^{(+)} ; p q\right\rangle+\left|b^{(+)} ; p q\right\rangle\right] \text {, } \\
& \left|\left(\omega_{1} \frac{3}{2}\right) \beta=2, H_{1}=p-q+\frac{1}{2}, T=M_{T}=\omega_{1}-1-p-q\right\rangle=\frac{2 \omega_{1}+1}{2\left(2 \omega_{1}+1-p-q\right)} \\
& \times N_{2}^{(+)}(p q)\left\{\frac{\left[\left(\omega_{1}-1-p-q\right)\left(2 \omega_{1}+5\right)-(q+1)\left(2 \omega_{1}-3-2 p-2 q\right)\right]}{q+1}\left|a^{(+)} ; p q\right\rangle\right. \\
& \left.+\frac{\left[\left(\omega_{1}-p-q\right)\left(2 \omega_{1}+5\right)-(p+1)\left(2 \omega_{1}-3-2 p-2 q\right)\right]}{3(p+1)}\left|b^{(+)} ; p q\right\rangle\right\}, \\
& N_{1}^{(+)}(p q)=(-1)^{p+q} \\
& \times\left\{\begin{array}{c}
-\frac{6(p+1)(q+1)\left(2 \omega_{1}+1-p-q\right)}{\left\langle a^{(+)} ; p q \mid b^{(+)} ; p q\right\rangle\left(2 \omega_{1}+1\right)} \\
\quad \times\left[\left(2 \omega_{1}+5\right)\left(\omega_{1}-p-q\right)(p+q+2)+(p+1)\left(2 \omega_{1}-3-2 p-2 q\right)\left(2 \omega_{1}+1-4 q\right)\right]
\end{array}\right\} \\
& N_{2}^{(+)}(p q)=N_{1}^{(+)}(p q) \\
& \times\left\{\frac{12(p+1)(q+1)\left(2 \omega_{1}+1-p-q\right)^{2}}{\left(\omega_{1}-p\right)\left(\omega_{1}+1-q\right)\left(2 \omega_{1}+1\right)\left(2 \omega_{1}+5\right)\left(2 \omega_{1}-3-2 p-2 q\right)\left(2 \omega_{1}+1-2 p-2 q\right)}\right\}^{\frac{1}{2}}, \\
& \left\langle a^{(+)} ; p q \mid b^{(+)} ; p q\right\rangle \\
& 3\left(2 \omega_{1}+1\right)\left(2 \omega_{1}-1-p-q\right)(p+1) !(q+1) !\left(2 \omega_{1}+2\right) ! \\
& =-\frac{\times\left(2 \omega_{1}\right) !\left(2 \omega_{1}-4\right) !\left(\omega_{1}-\frac{1}{2}-p\right) !\left(\omega_{1}+\frac{1}{2}-q\right) !}{\left(2 \omega_{1}-1\right) 2^{3 p+3 q-1}\left(2 \omega_{1}-2-2 p-2 q\right) !\left(\omega_{1}-\frac{1}{2}-p-q\right) !} . \\
& \times\left(\omega_{1}+\frac{1}{2}-p-q\right) !\left(2 \omega_{1}-2 p\right) !\left(2 \omega_{1}+2-2 q\right) !
\end{aligned}
$$


(ii) For those states with $\omega_{1}+\frac{3}{2}-T-H_{1}=$ odd integer the labels $\beta=-1,-2$ will be used (see table 3 ). Here define

$$
\begin{gathered}
\left|a^{(-)} ; p q\right\rangle=\frac{\sqrt{ } 2}{3\left(\omega_{1}+1\right)} O_{+1-1}^{p+1} O_{-1-1}^{q} O_{-10} O_{-20}|c . s t .\rangle \\
\left|b^{(-)} ; p q\right\rangle=\frac{\sqrt{ } 2}{\omega_{1}+1} O_{+1-1}^{p} O_{-1-1}^{q+1} O_{-10}|c . s t .\rangle
\end{gathered}
$$

where $H_{1}=p-q-\frac{1}{2}, T=\omega_{1}-1-p-q, y$ for this case is completely determined by (12) and the requirement of simple particle-hole conjugation symmetry. Thus,

$$
y^{(-)}\left(H_{1}, T\right)=-y^{(+)}\left(-H_{1}, T\right) .
$$

With this choice the normalized eigenstates become

$$
\begin{aligned}
& \left|\left(\omega_{1} \frac{3}{2}\right) \beta=-1, H_{1}=p-q-\frac{1}{2}, T=M_{T}=\omega_{1}-1-p-q\right\rangle \\
& =N_{1}^{(-)}(p q)\left[\left|a^{(-)} ; p q\right\rangle-\left|b^{(-)} ; p q\right\rangle\right], \\
& \left|\left(\omega_{1} \frac{3}{2}\right) \beta=-2, H_{1}=p-q-\frac{1}{2}, T=M_{T}=\omega_{1}-1-p-q\right\rangle=\frac{\left(2 \omega_{1}+1\right) N_{2}^{(-)}(p q)}{2\left(2 \omega_{1}+1-p-q\right)} \\
& \times\left\{\frac{\left[\left(\omega_{1}-1-p-q\right)\left(2 \omega_{1}+5\right)-(p+1)\left(2 \omega_{1}-3-2 p-2 q\right)\right]}{p+1}\left|a^{(-)} ; p q\right\rangle\right. \\
& \left.+\frac{\left[\left(\omega_{1}-p-q\right)\left(2 \omega_{1}+5\right)-(q+1)\left(2 \omega_{1}-3-2 p-2 q\right)\right]}{3(q+1)}\left|b^{(-)} ; p q\right\rangle\right\},
\end{aligned}
$$

where

$$
\begin{aligned}
& N_{1}^{(-)}(p q)=N_{1}^{(+)}(q p), \\
& N_{2}^{(-)}(p q)=N_{2}^{(+)}(q p) .
\end{aligned}
$$

\subsection{THE REPRESENTATION $(t+1, t)$}

All states of this representation are labeled $\beta=1,2$.

For those states with $T-H_{1}=$ odd integer define

$$
\begin{aligned}
|a ; m n\rangle= & \left|a ; H_{1} T\right\rangle=\frac{(t+2) 2^{m+1}}{(2 m+2) !} \\
& \times\left[2(m+1) O_{-1-1}^{n+1} O_{-20}^{m}-O_{-1-1}^{n} O_{-20}^{m+1} O_{+1-1}\right]|c . s t .\rangle, \\
|b ; m n\rangle= & \left|b ; H_{1} T\right\rangle=\frac{2^{m+1}}{(2 m+2) !} \\
& \times\left[2(t+1)(m+1) O_{-1-1}^{n+1} O_{-20}^{m}-(t-2 m) O_{-1-1}^{n} O_{-20}^{m+1} O_{+1-1}\right]|c . s t .\rangle,
\end{aligned}
$$

where $H_{1}=t-1-2 m-n, T=t-n$. 
For those states wiih $T-H_{1}=$ even integer define,

$$
\begin{aligned}
& \left|a ; H_{1} T\right\rangle=-\frac{\sqrt{2} 2^{m+1}}{(2 m+3) !} \\
& \times\left[-2(m+1) O_{-1-1}^{n+1} O_{-20}^{m} O_{-10}+O_{-1-1}^{n} O_{-20}^{m+1} O_{+1-1} O_{-10}\right]|\mathrm{c} . \mathrm{st} .\rangle, \\
& \left|b ; H_{1} T\right\rangle=-\frac{\sqrt{2} 2^{m+1}}{(t+2)(2 m+3) !} \\
& \left.\times\left[-2(t+2)(m+1) O_{-1-1}^{n+1} O_{-20}^{m} O_{-10}+(t-1-2 m) O_{-1-1}^{n} O_{-20}^{m+1} O_{+1-1} O_{-10}\right] \mid \text { c.st. }\right\rangle \text {, }
\end{aligned}
$$

with $H_{1}=t-2-2 m-n, T=t-n$.

For both cases, in order to guarantee simple conjugation properties, $y$ must be chosen to be odd. A choice of $y$ which makes the eigenstates simple is

$$
y\left(H_{1} T\right)=\frac{H_{1}(2 T+1)}{2(t+2+T)} .
$$

Then for all $H_{1}, T$ the normalized eigenstates are,

$$
\begin{aligned}
& \left|(t+1, t) \beta=1, H_{1}, T=M_{T}\right\rangle=-N_{1}\left(H_{1} T\right)\left|a ; H_{1} T\right\rangle, \\
& \left|(t+1, t) \beta=2, H_{1}, T=M_{1}\right\rangle \\
& =N_{2}\left(H_{1} T\right)\left\{-\frac{H_{1}(t+1)(2 T+1)}{T(T+1)(t+2+T)+H_{1}^{2}(t+1-T)}\left|a ; H_{1} T\right\rangle-\left|b ; H_{1} T\right\rangle\right\}, \\
& N_{1}\left(H_{1} T\right)=\left\{\overline{\left.\left\langle a ; H_{1} T\right| \overline{b ; H_{1}} \cdot \frac{H_{1}}{T\rangle[T+1)(2 T+1)}(\overline{T+1)(t+2}+T)+H_{1}^{2}(t+1-T)\right]}\right\}^{\frac{1}{2}}, \\
& N_{2}\left(H_{1} T\right)=\left\{\frac{H_{1}(t+2)(2 T+1)\left[T(T+1)(t+2+T)+H_{1}^{2}(t+1-T)\right]}{\langle a ;} \overline{H_{1}} \frac{T \mid b ; H,}{T\rangle(t+1-T)(t+2+T)\left(T^{2}-H_{1}^{2}\right)\left[(T+1)^{2}-H_{1}^{2}\right]}\right\}^{\frac{1}{2}}, \\
& \left\langle a ; H_{1} T \mid b ; H_{1} T\right\rangle \\
& =\frac{(t+1)^{3}(t+2)(2 t+3)^{2}\left(2 t^{2}-t+2\right) H_{1}\left(T+2-H_{1}\right)(2 t-1) !(t-T) !\left(2 t+3+H_{1}-T\right) !}{2^{2(t+1-T)}(2 T) !(t+2+T) !\left(t+H_{1}\right) !} .
\end{aligned}
$$

\section{Applications}

From the constructions given in sect. 3 it is possible to calculate the matrix elcments of the infinitesimal generators of $R(5)$, (see tables $A .1-3$ ), and from these the $\mathrm{R}(5)$ Wigncr cocflicients which give the $n, T$ dependent factors of one-particle fractional parentage coefficients (tables $\mathrm{A.4}$.7). Although these are sufficient to determine the $n, T$ dependence of all matrix elements of interest in nuclear physics, it will be more convenient to find the $n, T$ dependence of the matrix elements of the nucleon- 
nucleon interaction directly. It will be of particular interest to see under what conditions the degeneracy is removed for states of $T$ multiplicity 2 , that is states with the same $n, T$, and spatial charactcristics, but different $\beta$ labels, and to isolate those features of the actual interaction among nuclcons which are most effective in splitting the degeneracy of such isospiil doublets. The most important states with $T$ multiplicities of 2 are states with seniority $t=2$ and reduced isospin $t=1 ;(v=2$ is the lowest seniority for which $T$ multiplicitics of 2 can occur). These are the states of the irreducible representation $\left(\omega_{1} 1\right)$ with $\omega_{1}-T-H_{1}=2 j+1-T-\frac{1}{2} n-\frac{1}{2} v=$ odd integer. The matrix elements for such states are calculated in this section for (I) the most general charge independent (isoscalar) two-body interaction, and (ii) the Coulomb interaction, in a pure configuration $j^{n}$.

The decomposition of the two-body interaction into its $R(5)$ irreducible tensor components is giver in ref. $\left.{ }^{8}\right)$. These include the irreducible representations $\left(\omega_{1} t\right)=$ (22), (20), and (10). The necded R(5) Wigner cocfficients for the coupling with these representations are tabulated in ref. ${ }^{15}$ ), while the necessary reduced matrix elements for states with $v=2, t=1$ are given in ref. ${ }^{8}$ ). With these, the matrix elements of a two-body interaction can be expressed in terms of the two-particle matrix eicments

$$
V_{J T}=\left\langle j^{2} ; J M T M_{T}\left|V_{12}\right| j^{2} ; J M T M_{T}\right\rangle,
$$

and in terms of weighted averages over these. It is convenicnt to define

$$
\begin{aligned}
& V_{J}= \begin{cases}V_{J, T=0}, & J \text { odd } \\
V_{J, T=1}, & J \text { even, }\end{cases} \\
& \bar{V}_{\text {even }}=\frac{\sum_{\text {even } J} V_{J 1}(2 J+1)}{j(2 j+1)}, \quad \bar{V}_{\text {odd }}=\frac{\sum_{\text {odd } J} V_{J 0}(2 J+1)}{(j+1)} \overline{(2 j+1)}, \\
& \left.\overline{\left(V_{\text {eien }} U_{J_{2}}\right.}\right)=\frac{1}{j(2 j+1)} \sum_{\text {even } J} V_{J 1}(2 J+1) U\left(J_{j j} J_{2} ; j j\right), \\
& \left(\overline{V_{\text {odd }} U_{J_{2}}}\right)=\frac{1}{(j+1)(2 j+1)} \sum_{\text {odd } J} V_{.10}(2 J+1) U\left(J j j J_{2} ; j j\right) \text {. }
\end{aligned}
$$

The results for the isoscalar and Coulomb casses will be given separately.

\subsection{THE ISOSCALAR TW'O-BODY INTERACTION}

The matrix element which is off-diagonal in $\beta$ is

$$
\begin{aligned}
E_{12}\left(V_{J}\right)= & \left\langle\left(j-\frac{1}{2}, 1\right) \beta^{\prime}=1, I_{1} T M_{\mathrm{T}} ; J_{2} M_{J}|V|\left(j-\frac{1}{2}, 1\right) \beta=2, H_{1} T M_{T} ; J_{2} M_{J}\right\rangle \\
& \left.=-\frac{H_{1}}{3 j f\left(H_{1} T\right)}\left\{\frac{T(T+1)\left[\left(j+\frac{3}{2}+T\right)^{2}-I_{1}^{2}\right][}{\left(j+\frac{1}{2}\right)\left(j+\frac{3}{2}\right)} \underline{\left.-\frac{1}{2}-T\right)^{2}}-\underline{H_{1}^{2}}\right]\right\}^{\frac{1}{2}} \\
& \times\left\{\frac{(2 j+3)\left(j+\frac{1}{2}+3 T\right)}{(2 j-3)(2 j-5)}-\mathscr{C}-\mathscr{C}^{\prime}\right\} .
\end{aligned}
$$


The diagonal matrix elements are, with $\beta=1,2$

$$
\begin{aligned}
& E_{\beta \beta}=H_{1}\left\{3 j \bar{V}_{\text {even }}+(j+1) \bar{V}_{\text {odd }}\right\}+\frac{3}{20}\left\{(2 j-3) V_{0}+2(6 j+1) j \bar{V}_{\text {cven }}+4 j\left(\overline{V_{\text {even }} U_{J_{2}}}\right)+4 V_{J_{2}}\right\} \\
& +\frac{1}{5}\left\{2(j+1)^{2} \bar{V}_{\text {odd }}-(j+1)\left(\overline{\left(V_{\text {odd }} U_{J_{2}}\right.}\right)\right\} \\
& -\frac{\left(j+\frac{1}{2}\right)\left(j+\frac{3}{2}\right)-2 T(T+1)-4 H_{1}^{2}}{3(2 j-1)}\left\{\mathscr{A}-\frac{\left(j+\frac{1}{2}\right) \mathscr{C}}{2 j\left(j-\frac{3}{2}\right)\left(j-\frac{5}{2}\right)}\right\} \\
& +\frac{\left(j+\frac{1}{2}\right)\left(j+\frac{3}{2}\right)-5 T(T+1)+5 H_{1}^{2}}{60(j+1)}\left\{2 \mathscr{Q}^{\prime}-\frac{\mathscr{C}^{\prime}}{2 j\left(j+\frac{i}{2}\right)\left(j+\frac{3}{2}\right)}\right\}-\frac{\mathscr{C}^{\prime}}{30(j+1)} \\
& +\frac{(-1)^{\beta}}{j(2 j+1)}\left\{\frac{\mathscr{C}^{\prime}}{\left(j+\frac{3}{2}\right)}-\frac{\left(j+\frac{1}{2}+3 T\right) \mathscr{C}}{\left(j-\frac{3}{2}\right)\left(j-\frac{5}{2}\right)}\right\} \\
& \times\left\{\left(j+\frac{3}{2}+T\right)\left(j+\frac{1}{2}-T\right)+H_{1}^{2}-\frac{2 H_{1}^{2}\left(j+\frac{1}{2}\right)\left(j+\frac{3}{2}\right)(2 T+1)}{f\left(H_{1} T\right)}\right\}
\end{aligned}
$$

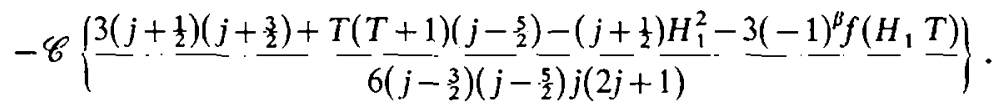

Here $f\left(H I_{1} T\right)$ is the function which appears in Wigner coefficients involving the representations $(\omega, 1)$

$$
f\left(H_{1} T\right)=(T+1)\left(j+\frac{3}{2}+T\right)\left(j+\frac{1}{2}-T\right)+T H_{1}^{2} .
$$

Also,

$$
\begin{aligned}
& \mathscr{A}=2\left[2 j \bar{V}_{\text {cren }}-V_{0}\right] \text {, } \\
& \mathscr{A}^{\prime}=j \bar{V}_{\mathrm{cven}}+V_{0}+3(j+1) \bar{V}_{\mathrm{odd}}, \\
& \mathscr{C}=2\left[2 j \bar{V}_{\text {even }}-V_{0}\right]+(2 j-1)\left[2 j\left(\overline{V_{\text {even }}} U_{J_{2}}\right)-V_{J_{2}}\right] \text {, } \\
& \mathscr{C}^{\prime}=j \bar{V}_{\text {even }}+V_{0}-2(j+1)\left[j\left(\overline{V_{\text {cven }}} U_{J_{2}}\right)+V_{J_{2}}\right]+3(j+1) \bar{V}_{\text {odd }}-6(j+1)^{2}\left(\overline{V_{\text {odd }} U_{J_{2}}}\right) .
\end{aligned}
$$

It is of interest to find the conditions under which the degeneracy of the isospin doublet $(\beta=1,2)$ is retained; that is the conditions on $V$, for which $\mathscr{C}=\mathscr{C}^{\prime}=0$, and consequently $E_{12}=0, E_{11}=E_{22}$. This condition is certainly satisfied for an interaction which is invariant in the five-dimensional quasispin space, that is an interaction built from odd (isoscalar) multipole moments only. For such an interaction

$$
V_{J T}=\sum_{k_{\text {usd }}} c_{k}(-1)^{T+1} \frac{U(j j j j ; k J)}{[2 J+1]^{\frac{U}{2}}} \text { with } c_{k} \text { arbitrary. }
$$

With such valites of $V_{J T}$ it can be seen that $\mathscr{C}=\mathscr{C}^{\prime}=0$. Another simple interaction 
which satisfies this condition is

$$
V^{(1)}=\left\{\begin{array}{l}
V_{J \text { cven }}^{(1)}=\bar{V}_{2}+J(J+1) \bar{V}^{\prime}, \quad \text { for } J \neq 0, \\
V_{J \text { odd }}^{(1)}=\bar{V}_{1}+J(J+1) \bar{V}^{\prime} \\
V_{0}^{(1)}=\text { arbitrary, } \\
\bar{V}_{2}, \bar{V}_{1}, \bar{V}^{\prime}=\text { arbitrary constants, }
\end{array}\right.
$$

that is, an interaction built from a combination of the following simple two-body operators: (a) $V_{i k}=1$, (b) $V_{i k}=j_{i} \cdot j_{k}$, (c) $V_{i k}=t_{i} \cdot t_{k}$, (d) $V_{i k}=$ isoscalar pairing interaction $=\sum_{M_{T}} A^{\dagger}\left(M_{T}\right) A\left(M_{T}\right)$. The eigenvalues of this simple interaction are a function of the quantum numbers $v, t, n, T$, and $J$ only and cannot remove the degeneracies associated with $T$ multiplicities in any irreducible representation of $R(5)$. However, this interaction is of some interest since it has many features in common with actual effective interactions which have been used to fit nuclear spectra in many regions of the periodic table. To illustrate this point the interaction $V^{(1)}$ is compared with a realistic effective interaction for the $1 f_{\ddagger}$ shell in table 5 . The

TABIE 5

Comparison of $V^{(1)}$ with realistic two-particle cnergics (MeV) for the $\mathrm{f}_{\bar{i}}$ shell

\begin{tabular}{|c|c|c|c|c|c|c|c|c|}
\hline--- & -- & $\begin{array}{l}V_{1}-V_{0} \\
---\end{array}$ & $\begin{array}{l}V_{2}-V_{0} \\
---\end{array}$ & $V_{3}-V_{0}$ & $V_{4}-V_{0}$ & $\begin{array}{c}V_{s}-V_{0} \\
---\end{array}$ & $\begin{array}{l}V_{6}-V_{0} \\
--\end{array}$ & $V_{7}-V_{0}$ \\
\hline Real ") & -3.09 & 0.69 & 1.51 & 1.98 & 2.76 & 2.32 & 3.53 & 0.55 \\
\hline$V^{(1)}$ & -3.09 & 0.90 & 1.5 & 1.75 & 2.7 & 3.25 & 4.5 & 5.5 \\
\hline
\end{tabular}

2) As an example of a rcalistic effective interaction, set $A$ of Diepcrink and Brussaard ${ }^{16}$ ) has been chosin. The simple intiraction $V^{(1)}$ of $c q$. (24) has been cvaluated with $V_{0}=-3.09, \Gamma_{1}=0.75$. $\bar{l}_{2}=-1.0, \overline{\gamma^{\prime}}:-0.083 \mathrm{MiV}$.

fit of the $V_{f}^{(1)}$ to the real two-particle encrgies is reasonably good with one very important exception: the two-body interaction in the state with $J=2 j$. (The real twobody interaction in the state with $J=2 j$ is almost as attractive as the interaction in the statc with $J=0$, whereas the model interaction $V^{(1)}$ is repulsive in a state with $J=2 j$.) This suggests that a modified interaction based on $V^{(1)}$ may serve as a relatively good model interaction provided an additional attractive term is added for the state with $J=2 j$. It appears that it is this term which is largely responsibie for the removal of the degeneracy associated with states of $T$ multipiicities greater than onc. If a modificd model interaction is introduced for which

$$
V_{j}^{(2)}=V_{J}^{(1)}+V^{\prime \prime} \delta_{, I, 2 j}
$$

the energy splitting of states with the same $n, T, M_{T}$, but different 4 th quantum number is given entirely by the parameter $l^{\prime \prime \prime}$. For the isospin doublets with $v=2$, 